\title{
Comparison of Anti-Platelet Efficacy of Four Different Marketed Formulations of Aspirin in Diabetic Patients
}

\author{
Authors \\ Kanharam Narayan Patel ${ }^{1}$, Bharti Ramchand Daswani ${ }^{2}$, \\ Balasaheb Baburao Ghongane ${ }^{3}$ \\ ${ }^{1}$ Junior Resident, ${ }^{2}$ Associate Professor, ${ }^{3}$ Professor and Head \\ Dept. of Pharmacology, B. J. Government Medical College and SGH Pune, India-411001 \\ Corresponding Author \\ Bharti Ramchand Daswani \\ Contact No.+91-9421965337, Email: daswani_bharti@ rediffmail.com
}

\begin{abstract}
Introduction: In India, Aspirin is marketed as multiple preparations for antiplatelet use and its drug release pattern is likely to affect efficacy.

Objectives: The study compares anti-platelet efficacy of four preparations of aspirin against ADP-induced platelet aggregability and bleeding time (BT); and assesses correlation between steady state plasma concentration (CSS) and antiplatelet effect of daily 150mg Aspirin.

Methods: This was a four armed, randomised, investigator-blinded, parallel-group, comparative study. Study was conducted in 121 subjects of Type 2 DM on oral anti-diabetics having one or more cardiovascular risk factors. Each subject was randomized to receive Plain aspirin, soluble, effervescent or enteric coated, for 15 days. Blood samples were taken at day 0 and day 15, two hours after dosing.

Results: Total 121 subjects completed study (Plain 30, soluble 31, effervescent 30 and enteric coated 30) and the baseline characteristics were similar among all groups. All preparations inhibited platelet aggregation (Reduction from day 0 to day 15: plain-25.9\%, soluble-23.9\%, effervescent-23.5\%, enteric coated-26.4\%; $p=0.89$ ) and increased BT (Rise: plain-236.4\%, soluble-236.1\%, effervescent-229\%, enteric coated-256.7\%; $p=0.87)$ to a similar extent. Salicylate CSS measured at day 15 was significantly higher in effervescent $(23.75 \mathrm{mg} / \mathrm{L})$ as compared to enteric coated $(19.73 \mathrm{mg} / \mathrm{L})$ and soluble $(19.27 \mathrm{mg} / \mathrm{L})$. Plain aspirin produced salicylate CSS of $20.8 \mathrm{mg} / \mathrm{L}$. There was no significant correlation between salicylate CSS and antiplatelet activity for study population [ $r=-0.124, p=0.179]$ or for individual groups.

Conclusions: -All preparations produced comparable antiplatelet effect which did not correlate with steady state plasma salicylate concentration at daily dose of $150 \mathrm{mg}$.

Keywords: Aspirin, Diabetes, Antiplatelet effect, Serum salicylate.
\end{abstract}

\section{Introduction}

Aspirin, otherwise known as acetylsalicylic acid (ASA), acts by acetylation of the two isoenzyme forms of prostaglandin $\mathrm{G} / \mathrm{H}$ synthase which results in the irreversible inactivation of the cyclooxygenase (COX) activity of this enzyme. The decreased platelet aggregation caused by aspirin is the most plausible mechanism for the cardioprotective effects of this drug ${ }^{[1]}$. 
Diabetes is one of the largest global health emergencies of the 21 st century ${ }^{[2]}$. Cardiovascular disease (CVD) is the major cause of morbidity and mortality for individuals with diabetes and is the largest contributor to the direct and indirect costs of diabetes ${ }^{[3]}$.

Diabetes mellitus is considered to be a prothrombotic state with chronic activation of the coagulation system and decreased fibrinolytic potential $^{[4]}$.

According to American diabetic association one should consider anti-platelet therapy (Aspirin 75$162 \mathrm{mg} /$ day) as a primary prevention strategy in those with type 1 or type 2 diabetes at increased cardiovascular risk ${ }^{[5]}$.

Currently there is wide range of aspirin preparations in the market across the world, including plain acetyl salicylic acid, effervescent tablet, enteric coated tablet, chewable tablet, soluble tablet, sustained release etc. available for both analgesic and antiplatelet effect. For example, aspirin's antiplatelet efficacy may be limited by coincidental inhibition of a prostacycline by fraction of aspirin reaching systemic circulation, after hepatic extraction. But Clarke R J et al. found that systemic synthesis of prostacyclin is preserved with a controlled-release formulation containing $75 \mathrm{mg}$ of aspirin ${ }^{[6]}$. But it may not be same with other preparations. Each of the marketed preparations uses a different manufacturing technology and that in vivo pharmacokinetics and pharmacodynamics may vary with change in in vitro drug release profile and so does the anti-platelet activity. Considering this, it is apparently essential that each type of aspirin preparation undergoes adequate comparison study for their antiplatelet activity.

Additionally, the disease related predisposing factors may introduce a variability in the antiplatelet activity of aspirin, for example, platelets in diabetes are more reactive and are turned over more rapidly than platelets from normal individuals ${ }^{[7]}$. In order to reduce the impact of this variability on the results, this study was conducted in patients with diabetes mellitus with risk factors rather the general population requiring antiplatelet therapy.

\section{Aim and Objectives}

So primary objective of study was to assess and compare the anti-platelet effect of following four different marketed preparations of Aspirin (administered orally) in diabetic patients with at least one more cardiovascular risk factor by studying ADP-induced platelet aggregation ex vivo. Secondary objectives were to find out if there is a co-relation between serum salicylate concentration at steady state and its anti-platelet activity and to study the disintegration time and dissolution rate of aspirin preparations to be used.

\section{Methods}

This study is a single centre, four armed, randomised, investigator-blinded, parallel-group, and comparative clinical study. The study was conducted from October 2014 to July 2016 in a tertiary care hospital where patients attending the diabetic outpatient department (OPD) were recruited for the study.

The study protocol, including Subject Information and Consent Form, was approved by the Ethics Committee of the tertiary care institute before enrolment of any subject into the study. The study was performed according to guidelines of Good Clinical Practice.

In a pilot evaluation, it was observed that $150 \mathrm{mg}$ of conventional uncoated formulation of aspirin inhibited platelet aggregation by around $25 \%$. A total sample size of 100 would be required to detect a difference of $33 \%(\alpha$ error $=0.05$, power $=80 \%$ ) among different formulations. Hence considering $15-20 \%$ drop out 120 subjects (30 in each group) were selected ${ }^{[8]}$.

\section{Inclusion Criteria}

Male patients with Type $2 \mathrm{DM}$, receiving oral antidiabetic (metformin 0.5-2 g/day and sulfonylurea: glimepiride 1-2 g/day) since at least 4 weeks and who had one or more other risk factors for cardiovascular disease (CVD) (Hypertension, Tobacco use, Diabetes, Obesity, Physical Inactivity, Unhealthy Diet, Raised cholesterol, Male sex, Advancing Age and Family History) ${ }^{[9]}$ were selected. 


\section{Exclusion Criteria}

Patients having age $<40$ years uncontrolled diabetes mellitus (Fasting Plasma Glucose Level> 300 $\mathrm{mg} / \mathrm{dL}$ despite therapy, requiring insulin, uncontrolled hypertension, abnormal liver function tests and/or renal function tests, receiving any form or dose of Aspirin therapy for $>7$ days, receiving any other antithrombotic or anti-coagulant therapy, or deranged bleeding and/ or clotting time at baseline study were excluded from the study.

Prior to inclusion in the study, subjects were given all information and details about the study in vernacular language and English. A written informed consent was obtained from each subject.

\section{Study Groups}

Group 1: Tablet Acetyl Salicylic Acid-ASA (Uncoated Tablet Aspirin I.P. $300 \mathrm{mg}$ ) (Scored tablet, mean weight of 10 half tablets was $156.3 \pm$ $2.8 \mathrm{mg}$ and that of 5 Full tablets was $315.4 \pm 1.3$ $\mathrm{mg}$ ). $1 / 2$ tablet daily in the morning on empty stomach for 15 days.

(Batch no. 10080, Mfg. Date- 12/2014, Exp. Date11/2016, Available in pharmacy of Tertiary Care Hospital, MFD by: Penta Pharmaceuticals, Mumbai, India.)

Group 2: Tablet Aspisol (Soluble Uncoated Tablet $150 \mathrm{mg}$ Aspirin I.P. $+75 \mathrm{mg}$ Glycine I.P.) $150 \mathrm{mg}-1$ tablet daily in the morning on empty stomach for 15 days.

(Batch no. R14451112, Mfg. Date- 12/2014, Exp. Date- 11/2016, MFD by: Shreya Lifesciences, Uttarakhand, India)

Group 3: Tablet Colsprin (Effervescent Uncoated Tablet - Aspirin I.P. 100 mg) (Scored tablet, mean weight of 10 half tablets was $83.7 \pm 3.9 \mathrm{mg}$ and that of 5 Full tablets was $156.8 \pm 2.2 \mathrm{mg}$ ) $-11 / 2$ tablet daily in the morning on empty stomach for 15 days. Patient was asked to place the tablet in a table spoon $(15 \mathrm{ml})$ and add water to fill it fully, allow the tablet to dissolve completely, then drink all of the liquid.

(Batch no. A105, Mfg. Date- 04/2014, Exp. Date02/2017, MFD by: Reckitt \& Benckiser (India) Ltd, New Delhi, India.)
Group 4: Tablet Ecosprin (Enteric coated Tablet Aspirin I.P. $150 \mathrm{mg}$ ) -1 tablet daily in the morning on empty stomach for 15 days.

(Batch no. 04005901, Mfg. Date- 02/2015, Exp. Date- 01/2017, MFD. By: USV Ltd., Bangalore, India.)

The drug was dispensed as per computer generated randomization code in opaque packets. The investigator was not aware of the formulation issued to the subject.

\section{Detailed Research Plan}

OPD Screening: In diabetic OPD patients were selected on the basis of clinical inclusion and exclusion criteria.

Visit 1 (Day -1):Subjects found clinically suitable for the study, underwent physical examination and the biochemical tests viz. fasting plasma glucose level (FPGL), clotting time (CT), prothrombin time (PT), liver function tests (LFT), renal function test (RFT) and lipid profile.

Fasting blood was collected 8am to 10am.

Subjects found fit according to study criteria were called next day (Day 0).

Visit 2 (Day 0): A written informed consent (WIC) were obtained from volunteering patients.

Blood sample was collected for baseline platelet aggregability study (PAD0) and bleeding time (BTD0). Subjects were randomized to one of the treatment groups as mentioned above. Subjects were given sealed packets of medication along with their oral hypoglycaemic drugs and were instructed to take daily dose of drug every day before breakfast for 15 days starting from next day (Day 1). They were counselled about the importance of taking daily dosage of the drug prescribed and its effects and possible side effects.

Phone call 1 (Day 1): Subject was asked about first dose and reminded again about the importance of taking daily dosage of the drug prescribed and its effects and possible side effects.

Phone call 2 (Day 8): Every subject was followed up for any adverse effect and enquiry about compliance. 
Visit 3 (Day 15):The every patient was called, after taking his Day 15 dose of drug, for clinical examination, adverse effects, if any and blood sample collection for determining platelet aggregability (PAD15), BT (BTD15)and steady state serum salicylate concentration (CSS). Blood was collected 2 hours after of dosing.

All data from laboratory tests and other investigations was entered promptly, in accordance with specific instructions accompanying the case record forms (CRF). The subjects were questioned about occurrence of the adverse events on day 1 and day 8 by telephone; and also on day 15 i.e. visit 3 .

\section{Study Parameters}

Comparison between the four formulations with regard to

1. Platelet aggregability at Day 0 (PAD0) and Day 15 (PAD15)

Platelet aggregation was estimated by the colorimetric method (Filter Colorimeter, Model No.: H0391, Date: 08/06/2001, by Kanad Vidyut, Pune.) as given by O'Brien J.R. ${ }^{[10,11]}$.

Principle: "Absorbance of platelet rich plasma (PRP) is directly proportional to the concentration of platelets in it".

When ADP is added to the constantly stirred PRP, it induces platelet aggregation and forms clumps. The clumps settle down to the bottom of the test tube and so there occurs a decrease in the absorbance of plasma. More the platelet aggregability less is the absorbance.

2. BT at Day 0 (BTD0) and Day 15 (BTD15)

BT was determined by Duke's filter paper method [12].

3. Steady state serum salicylate level at Day15 (CSS) Steady state serum salicylate level at day 15(CSS) was estimated by Trinder's rapid method ${ }^{[13]}$.

Principle: Salicylate ion when combined with ferric salts in weakly acid solution gives a purple colour. This purple colour is proportional to the concentration of salicylates and is used for the determination of salicylates in serum by the colorimetric method (Filter Colorimeter, Model No.: H0391, Date: 08/06/2001, by Kanad Vidyut, Pune.).
Ferric Nitrate + Sodium Salicylate $===>$ Ferric Salicylate + Sodium Nitrate

$\mathrm{Fe}(\mathrm{NO} 3) 3+3(\mathrm{C} 7 \mathrm{H} 5 \mathrm{NaO} 3)===>\mathrm{Fe}(\mathrm{C} 7 \mathrm{H} 5 \mathrm{O} 3) 3+$ 3(NaNO3)

To eliminate any inhibition of this colour by phosphates or oxalates, a reagent was prepared containing a high concentration of ferric nitrate. Mercuric chloride and hydrochloric acid were incorporated into the solution in order to precipitate the protein in the serum.

4. Disintegration Time and Dissolution Rate

The Disintegration time and Dissolution rate of the four formulations were studied at gastric $\mathrm{pH}$ for all preparations of aspirin and also at intestinal $\mathrm{pH}$ for enteric coated aspirin ${ }^{[14,15,16]}$.

\section{Statistical Analysis}

Data entry and analysis was done by principal investigator.

Change in the platelet aggregability and BT from baseline were compared between different groups by ANOVA test.

Statistical tests was applied to evaluate correlation between plasma salicylate concentration at steady state and its anti-platelet activity (i.e. change in platelet aggregability from baseline).

Microsoft Excel 2013 was used to calculate all statistical tests.

\section{Results}

A total of 134 subjects were enrolled in this study. Each was randomly assigned to receive $150 \mathrm{mg}$ of one of four formulations of aspirin viz. Plain aspirin, Aspisol (soluble), Colsprin (effervescent) and Ecosprin (enteric coated). Out of these, 13 were lost in follow-up and 121 completed study (Plain 30, Aspisol 31, Colsprin 30 and Ecosprin 30) and thus included for analysis.

None of the baseline characteristics showed statistically significant difference and were comparable in all four groups. 
Figure 1: Flow chart of study progression and number of subjects involved.

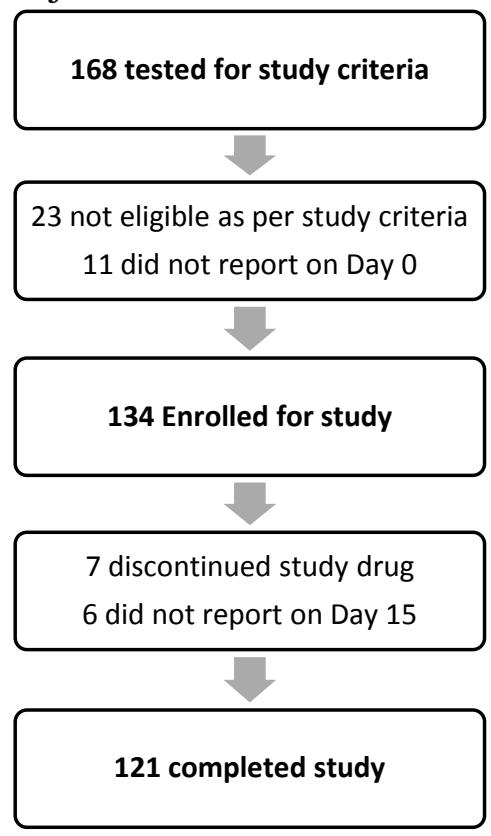

Thirty six subjects were only on metformin [0.5-2 gm/day] (Plain 8, Aspisol 9, Colsprin 10 and Ecosprin 9) and 85 were on metformin and sulfonylurea [glimepiride 1-2 mg/day] (Plain 22, Aspisol 22, Colsprin 20 and Ecosprin 21).

\section{Antiplatelet activity (Figure 2):}

Platelet aggregation was measured on day 0 (PAD0) i.e. a day before first dose and at 2 hours after the last day dose (PAD15). Antiplatelet activity was calculated in terms of percentage change in platelet aggregation $(\% \triangle \mathrm{PA})$ from Day $0(\mathrm{PAD} 0)$ to Day 15 (PAD15)

Hence, $\% \Delta \mathrm{PA}=(\mathrm{PAD} 15-\mathrm{PAD} 0) * 100 / \mathrm{PAD} 0$.

All preparations showed negative (-) change in platelet aggregation (\% $\%$ PA: Plain: -25.9 , Aspisol: 23.9, Colsprin: -23.5, Ecosprin: -26.4) i.e. they inhibited platelet aggregation. All four preparations inhibited platelet aggregation to a similar extent ( $\mathrm{p}$ value by $A N O V A=0.89$ ).
Figure 2: Comparison of percentage change in platelet aggregation $(\% \Delta \mathrm{PA})$ in each group

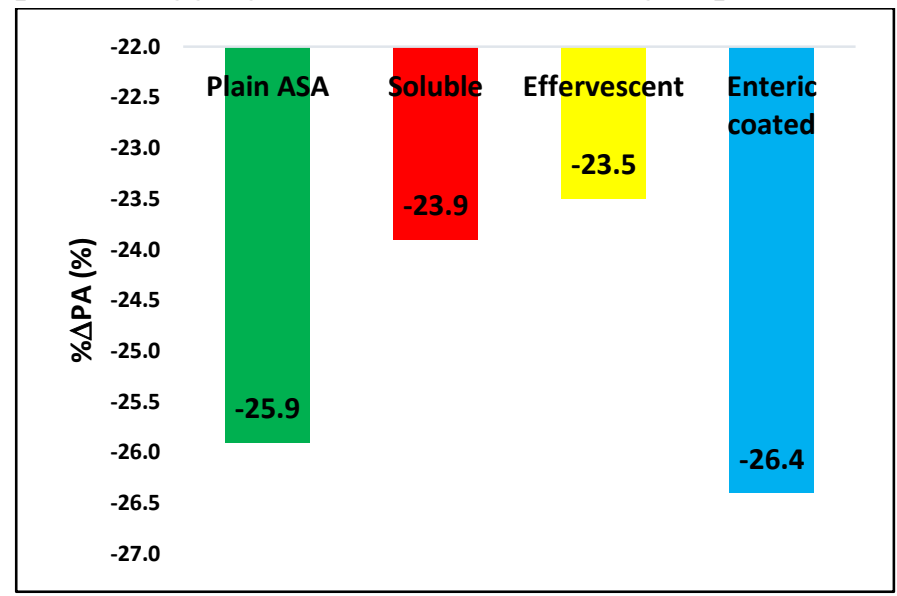

\section{Bleeding Time (BT) (Figure 3):}

Bleeding time (BT) was measured on day 0 (BTD0) i.e. a day before first dose and at 2 hours after the last day dose (BTD15). Percentage change in bleeding time $(\% \Delta \mathrm{BT})$ from Day 0 to Day 15 were calculated as-

$\% \Delta \mathrm{BT}=(\mathrm{BTD} 15-\mathrm{BTD} 0) * 100 / \mathrm{BTD} 0$.

All preparations increased BT $(\% \Delta \mathrm{BT}$ : Plain: 236.4\%, Soluble: 236.1\%, Effervescent: 229\%, Enteric coated: $256.7 \%$ ) to a similar extent (pvalue by ANOVA=0.87).

Figure 3: Comparison of percentage change in Bleeding Time $(\% \Delta \mathrm{BT})$ in each group.

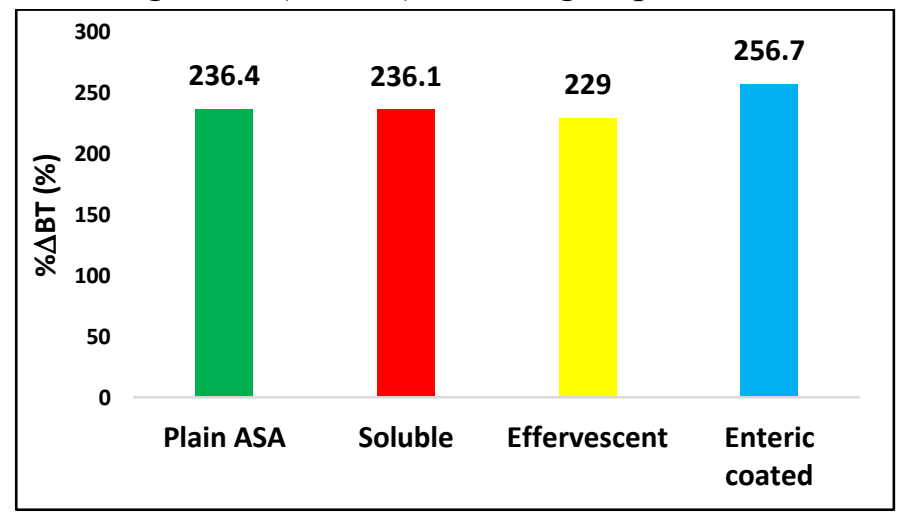

Correlation between steady state plasma salicylate concentration and anti-platelet activity: Steady state serum salicylate level (CSS), measured at 2 hours after last day dose i.e. day 15 was compared among all groups using ANOVA and Unpaired' $t$ ' test. It was found to be significantly higher with effervescent $(23.75 \mathrm{mg} / \mathrm{L})$ as compared 
to enteric coated $(19.73 \mathrm{mg} / \mathrm{L})$ and soluble (19.27mg/L). With plain ASA, it was $20.8 \mathrm{mg} / \mathrm{L}$.

There was no correlation between steady state plasma salicylate concentration and anti-platelet activity (percentage inhibition of platelet aggregation $(\% \Delta \mathrm{PA})$ for entire study population (coefficient of correlation $(r)=0.124, p=0.179)$ as well as individual groups. Coefficient of correlation (r) and Level of Significance (p) values were as follows: Plain ASA (-0.186, 0.326), soluble (0.21, $0.257)$, effervescent $(0.18,0.344)$, enteric coated (0.245, 0.194). (Figure 4)

Also there was no correlation between steady state plasma salicylate concentration and percentage change in bleeding time $(\% \Delta \mathrm{BT})$ for entire study population as well as individual groups.

Figure 4: Correlation between steady state plasma salicylate concentration (CSS) and anti-platelet activity (percentage change in platelet aggregation $(\% \Delta \mathrm{PA})$

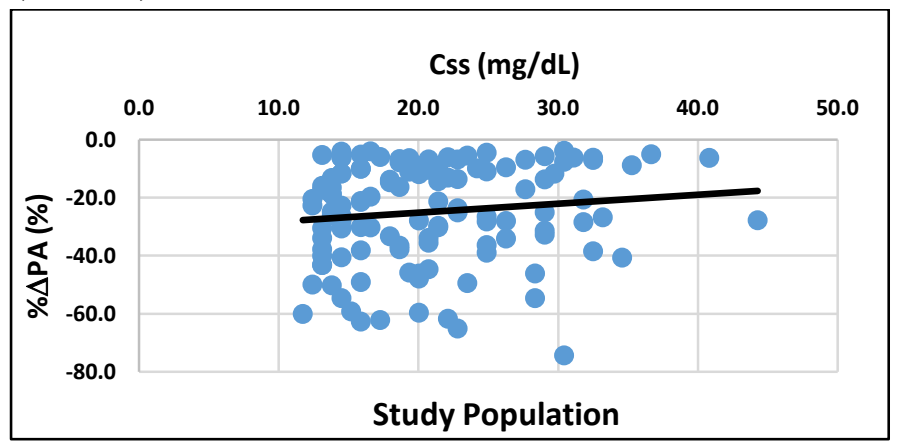

\section{Disintegration and dissolution data}

Six tablets of each formulations were tested for disintegration and dissolution studies.

Disintegration Time: There was a significant difference in the disintegration time of all aspirin formulations. The effervescent formulation showed fastest disintegration ( $29 \pm 8$ seconds) followed by plain ASA (41 \pm 4.3 seconds) then Soluble ASA (342 \pm 71 seconds) and slowest with enteric coated $(2313 \pm 287$ seconds, counted after $120 \mathrm{~min}$ of acetate buffer).

Dissolution Rate: ANOVA showed significant difference among all groups at all-time points. The enteric coated tablet dissolved only $4.6 \%$ until $\mathrm{pH}$ of the medium made alkaline (i.e. after 120 minutes).
Therefore difference between enteric coated aspirin and other formulations was very evident. Among the remaining 3 formulations, effervescent formulation showed greater dissolution rate as compared to plain ASA up to $50 \mathrm{~min}$. Soluble aspirin showed lesser dissolution than plain ASA up to $60 \mathrm{~min}$. The difference between dissolution of effervescent and soluble aspirin remained significant up to $80 \mathrm{~min}$. By 90 minutes $92.3 \pm 3.9 \%$ plain ASA, $90.8 \pm 4.5 \%$ soluble aspirin and $94.9 \pm$ $4.1 \%$ effervescent aspirin dissolved. Enteric coated aspirin showed $93.2 \pm 4.1 \%$ by 210 minutes i.e. 90 minutes after being placed in alkaline medium.

\section{Discussion}

All preparations inhibited platelet aggregation and increased BT to a similar extent.

No similar study has been done so far, to compare different Indian formulations of aspirin $150 \mathrm{mg}$ for antiplatelet efficacy in terms of inhibition of platelet aggregation and change in bleeding time. But some studies have evaluated different other preparations of different dosages.

Gatti $\mathrm{G}$ et al. compared formulation of soluble aspirin (l-ornithine acetylsalicylate) with conventional tablets and two other soluble dosage forms ( $d, 1$-lysine acetylsalicylate and a buffered effervescent formulation of acetylsalicylic acid). As compared to the soluble formulations, acetylsalicylic acid given as tablets resulted in slower absorption ${ }^{[17]}$.

Cox D et al. compared enteric-coated 75-mg aspirins with dispersible aspirin $75 \mathrm{mg}$, in healthy volunteers. Enteric coated preparation preparations tested were inferior to dispersible aspirin in their effect on serum thromboxane B2 level ${ }^{[18]}$. The effect of a formulation of enteric coated, low-dose (81 mg) aspirin on serum generated thromboxane B2 and platelet aggregation in healthy subjects were assessed by Vacken AV et al in a placebo-controlled study. The anti-platelet effect of multiple, daily, low-dose aspirin was not adversely affected by enteric coating ${ }^{[19]}$.

Cruz et al. reported that the pharmacodynamic profile of the extended release (ER) formulations 
was better than that of plain formulated aspirin in terms of thromboxane/prostacyclin balance and nitric oxide production ${ }^{[20]}$.

In present study there was no significant correlation between steady state plasma salicylate (SA) concentration within range of 19.27 to $23.75 \mathrm{mg} / \mathrm{dL}$ and anti-platelet activity for study population [ $\mathrm{r}=$ $0.124, p=0.179$ ] or for individual groups.

It could be explained by two ways. Firstly aspirin or ASA is responsible for antiplatelet activity by virtue of acetylation of COX enzyme in portal circulation, rather than SA. Thus, apparently, lack of correlation between SA and antiplatelet activity reflects inability of SA to inhibit platelets. Alternatively, because ASA gets deacetylated within 30 min to SA, ASA level cannot be measured, SA is a surrogate marker of bioavailability of ASA. Thus, a lack of correlation between steady state concentration of SA of 19.27 to $23.75 \mathrm{mg} / \mathrm{dL}$ and antiplatelet activity could be a reality suggesting that irrespective of the manner in which ASA is released from the formulation, the amount released produces similar antiplatelet activity.

Steady state serum salicylate level was estimated 2 hours after the last dose because the CSS gives a fair idea about the range in which the serum concentration is expected to lie after multiple doses.

\section{Conclusion}

Present study compared four marketed preparations of aspirin for antiplatelet efficacy in terms of change in platelet aggregation and bleeding time. It was found that all four have comparable antiplatelet efficacy. The study also evaluated the correlation of steady state plasma salicylate level with antiplatelet effect, which was found to be statistically insignificant within the observed range of plasma concentrations at a daily dose of $150 \mathrm{mg}$ Acetyl salicylic acid.

\section{References}

1. Farrugia CA. The antiplatelet activity of aspirin. The chronic ill. 1999;3:14-18 URL:http://www.mcppnet.org/publications/I SSUE03-5.pdf Accessed on: October 6,
2016 9:01:37 PM Executive Summary. In: International Diabetes Federation, 7 ed. Brussels, Belgium: International Diabetes Federation, 2015 p.12-20

URL:http://www.diabetesatlas.org Accessed on: February 03 2016, 1:29:13PM

2. Ali MK, Bullard KM, Saaddine JB, Cowie CC, Imperatore G, Gregg EW. Achievement of goals in U.S. diabetes care, 1999-2010. N Engl J Med 2013;368:1613-1624

URL:http://globalhealth.emory.edu/resource s/scholarly_publications/pdfs/2013_achieve mentofgoals_NEJM.pdf Accessed on: October 6, 2016 9:11:27 PM

3. Carr ME. Diabetes mellitus: A hypercoaguable state. J diabetes Complications 2001;15:44-54.

URL:https://www.ncbi.nlm.nih.gov/pubmed /11259926 Accessed on: October 6, 2016 9:12:21 PM

4. American Diabetes Association. Cardiovascular Disease and Risk Management. Diabetes Care 2016;39(Suppl. 1):S60-S71

5. URL:http://care.diabetesjournals.org/content /supp1/2015/12/21/39.Supplement_1.DC2/20 16-Standards-of-Care.pdf Accessed on:February 03, 2016, 1:43:13 PM

6. Clarke R. J., Mayo G., Price P., FitzGerald G. A. Suppression of thromboxane A2 but not of systemic prostacyclin by controlledrelease aspirin. $\mathrm{N}$ Engl $\mathrm{J}$ Med. 1991;325(16):1137-1141.

URL:http://www.nejm.org/doi/full/10.1056/ NEJM199110173251605\#t=article Accessed on: October 6, 2016 8:12:23 PM.

7. De BerardisG,Michele S, Giovanni S F M, Fabio P, Giusi G, Gianni Tet al Aspirin for primary prevention of cardiovascular events in people with diabetes: meta-analysis of randomised controlled trials. BMJ 2009;339:4531.

8. Whitley E, Ball J Statistics review 4: sample size calculations. Crit Care. 2002;6(4):33541. 
9. Mendis S, Puska P, Norrving B editors. Cardiovascular disease (CVD) Backgrounder - Epresspack- Global Atlas on Cardiovascular Disease Prevention and Control. World Health Organization (in collaboration with the World Heart Federation and World Stroke Organization), Geneva 2011.

10. O'Brien J R. Platelet aggregation. II. Some results from a new method of study. $\mathbf{J}$ ClinPathol. 1962;15:452-481.

11. 0' Brien JR. A comparison of platelet aggregation produced by seven compounds and comparison of their inhibitors. J Clinical Path 1964;17:275-281.

12. Pal GK, Pal P. Textbook of Practical Physiology, 3rd edn. 2010. p.100-01

13. TrinderP. Rapid determination of salicylates in biological fluids: Biochemical J. 1954; 54; 301.

14. Khan F., Li M., Schlindwein W. Comparison of In Vitro Dissolution Tests for Commercially Available Aspirin Tablets. Dissolution Technologies 2013;20(1):48-58

15. Appendix 7: Disintegration and Dissolution Tests. Indian Pharmacopoeia. 1996 Vol II. Delhi: The Controller of Publications; 1996. p. 734-46, A-80-84.

16. Opadry Enteric -94 Series, Preparation and Use Guidelines. Colorcon Technical Literature. Ads_Opadry Enteric Aspirin_V1_12.2009

URL:https://www.colorcon.com/literature/m arketing/mr/Delayed\%20Release/Opadry\%2 0Enteric/Chinese/ads_opadry\%20enteric_as pirin_FINAL_02.09.10.pdf Accessed on 8th August 2016; 04:32:12 pm

17. Gatti G, Barzaghi N, AttardoParrinello G, Vitiello B, Perucca E. Pharmacokinetics of salicylic acid following administration of aspirin tablets and three different forms of soluble aspirin in normal subjects. Int $\mathbf{J}$ ClinPharmacol Res. 1989;9(6):385-9.
18. Cox D, Maree AO, Dooley M, Conroy R, Byrne MF and Fitzgerald DJ. Effect of Enteric Coating on Antiplatelet Activity of Low-Dose Aspirin in Healthy Volunteers. Stroke 2006;37:2153-2158.

19. Van Hecken A, Juliano ML, Depre' M , De LepeleireI, Arnout J, Dynder A et al. Effects of enteric-coated, low-dose aspirin on parameters of platelet function. Aliment PharmacolTher 2002; 16: 1683-1688.

20. Cruz, J. P. De, Guerrero A., Gonza, J. A. Effects of two preparations of 75-mg extended-release aspirin on platelet aggregation, prostanoids and nitric oxide production in humans, 131568. European Journal of Clinical Pharmacology.2002; 11(57): 775-780. 